\title{
Early Expression of Odorant Receptors Distorts the Olfactory Circuitry
}

\author{
Minh Q. Nguyen, ${ }^{1}$ Carolyn A. Marks, ${ }^{2,3}$ Leonardo Belluscio, ${ }^{2}$ and Nicholas J. P. Ryba ${ }^{1}$ \\ ${ }^{1}$ Laboratory of Sensory Biology, National Institute of Dental and Craniofacial Research, National Institutes of Health (NIH), and ${ }^{2}$ Development Neural \\ Plasticity Unit, National Institute of Neurological Diseases and Stroke, NIH, Bethesda MD 20892, and 3Division of Molecular Neurobiology, Department of \\ Neuroscience, Karolinska Institute, SE-171 77 Stockholm, Sweden
}

The odor response properties of a mammalian olfactory sensory neuron (OSN) are determined by the tightly regulated expression of a single member of a very large family of odorant receptors (ORs). The OR also plays an important role in focusing the central projections of all OSNs expressing that particular receptor to a pair of stereotypic locations (glomeruli) in each olfactory bulb (OB), thus creating a spatial map of odor responses in the brain. Here we show that when initiated late in neural development, transgenic expression of one OR in almost all OSNs has little influence on the architecture of the OB in mice. In contrast, early OR-transgene expression (mediated by the G $\gamma 8$-promoter) in 50-70\% of OSNs grossly distorts the morphology of glomeruli and alters the projection patterns of many residual 0SNs not expressing the transgene. Interestingly, this disruption of targeting persists in adult animals despite the downregulation of $\mathrm{G} \gamma 8$ and transgenic OR expression that occurs as olfactory neurogenesis declines. Indeed, functional imaging studies reveal a dramatic decrease in the complexity of responses to odorants in adult $\mathrm{G} \gamma 8$-transgenic $\mathrm{OR}$ mice. Thus, we show that initiation of transgenic OR expression early in the development of OSNs, rather than just the extent of transgene expression, determines its effectiveness at modifying $\mathrm{OB}$ anatomy and function. Together, these data imply that OR-expression timing needs to be very tightly controlled to achieve the precise wiring and function of the mammalian olfactory system.

\section{Introduction}

Odorant receptors (ORs) are encoded by $\sim 1300$ different genes in mice (Buck and Axel, 1991; Zhang and Firestein, 2009) and are selectively expressed in the olfactory sensory neurons (OSNs) of the main olfactory epithelium (MOE). It has been demonstrated that each OSN expresses just one OR allele, setting the odorant selectivity of each neuron (Chess et al., 1994; Malnic et al., 1999). ORs also play an instructive role in guiding the central projection of OSNs to specific glomeruli (Ressler et al., 1994; Vassar et al., 1994; Mombaerts et al., 1996; Wang et al., 1998) and the establishment of a chemotopic map in the olfactory bulb (OB) (Malnic et al., 1999; Rubin and Katz, 1999; Belluscio and Katz, 2001).

The use of molecular genetic techniques to swap OR genes (e.g., the expression of one OR from the locus that normally drives a different receptor) demonstrated the role of ORs in determining OSN projection to specific glomeruli (Mombaerts et al., 1996; Wang et al., 1998). However, complex mechanisms controlling OR gene expression have prevented the use of straight-

Received March 24, 2010; revised May 4, 2010; accepted May 28, 2010.

This research was supported by the intramural research program of the National Institutes of Health and National Institute of Dental and Craniofacial Research (N.J.P.R.), and National Institute of Neurological Disorders and Stroke (L.B.). We thank Mark Hoon and members of our laboratories for valuable comments. M.Q.N. designed the study, performed experiments, analyzed data, and wrote the manuscript; C.A.M. performed experiments and analyzed data; L.B. designed the study and analyzed data; and N.J.P.R. designed the study, analyzed data, and wrote the manuscript.

Correspondence should be addressed to Minh Q. Nguyen, Building 49, Room 1A16, 49 Convent Drive, Bethesda, MD 20892. E-mail:mn143p@nih.gov.

DOI:10.1523/JNEUROSCI.1502-10.2010

Copyright $\odot 2010$ the authors $\quad$ 0270-6474/10/309271-09\$15.00/0 forward transgenic methods to manipulate their expression. Recently, we and others developed a tetracycline transactivator (TTA)-based approach to broadly express a single TetO-OR transgene in many OSNs throughout the MOE (Nguyen et al., 2007; Fleischmann et al., 2008). Remarkably, expression of a transgenic OR (tgOR) in an OSN completely suppressed expression of nontransgenic ORs (ntORs) in that neuron. However, when a single OR was expressed in $>90 \%$ of mature OSNs (Fleischmann et al., 2008), surprisingly little change in the anatomy or organization of the $\mathrm{OB}$ occurred (including targeting of residual ntORexpressing OSNs). We envisioned that two possibilities might explain this phenotype: either ORs play a less important instructive role than previously suggested or expression of TetO-ORs occurred too late in OSN development to influence their projections to the $\mathrm{OB}$.

OR expression in the developing olfactory system occurs by embryonic day 12.5, long before the first OSN synaptic connections are established (Sullivan et al., 1995). However, olfactory development is particularly prominent immediately after birth, with glomeruli and the glomerular map forming in the first 3 weeks of life (Potter et al., 2001). Even after the olfactory system has matured, OSNs continue to turnover at a lower rate and a variety of molecular markers, including growth-associated protein 43 (GAP43), G $\gamma 8$, the odorant gated channel, and olfactory marker protein (OMP), have established a stratified representation of OSN developmental stages in the MOE (Rogers et al., 1987; Ryba and Tirindelli, 1995; Munger et al., 2001; Cheng and Reed, 2007). The most immature cell bodies express GAP43 and occupy basal positions. As OSNs mature, their cell bodies migrate 

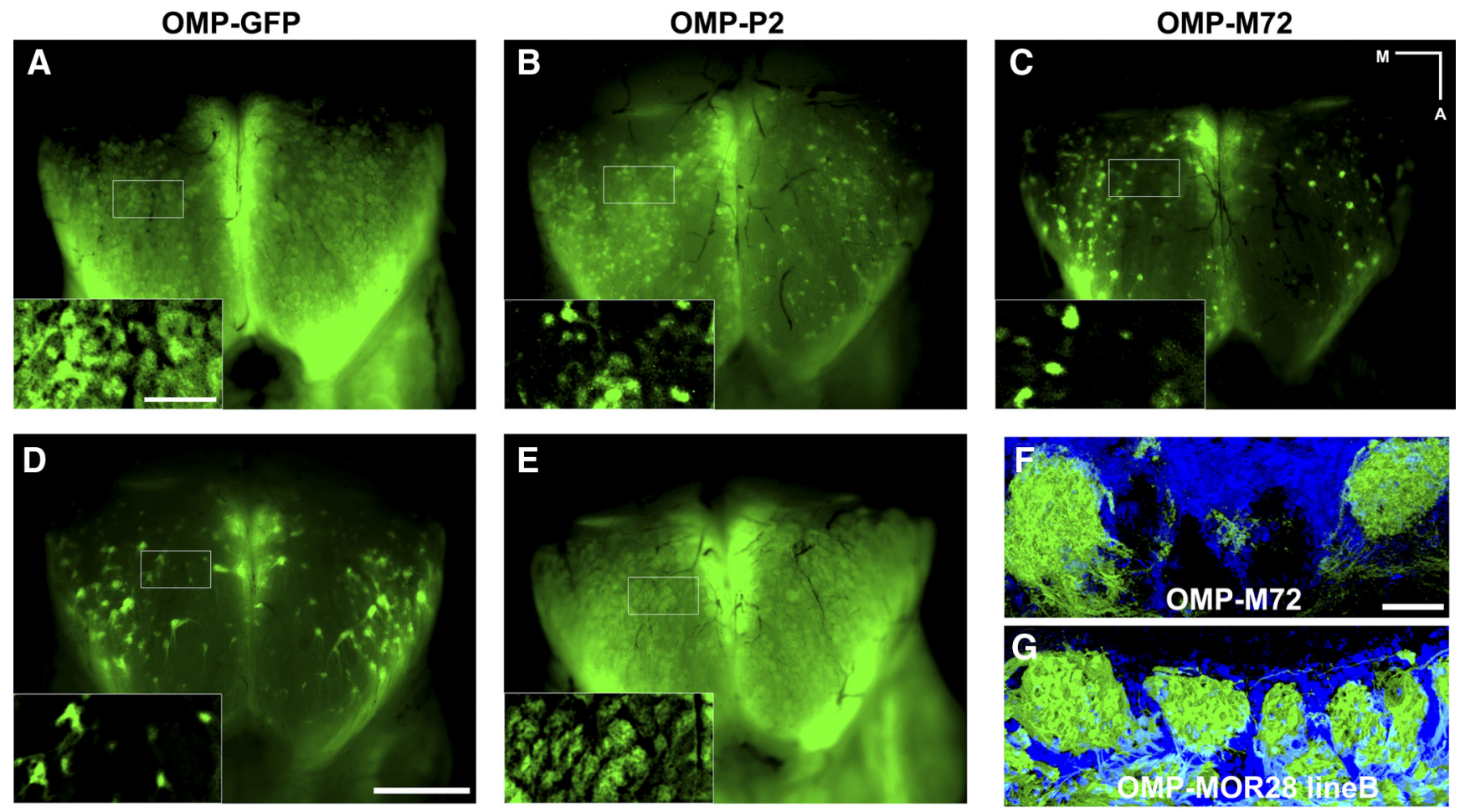

OMP-rl7

OMP-MOR28 line B

Figure 1. Projection patterns of OSNs expressing OMP-tgORs. $\boldsymbol{A}-\boldsymbol{E}$, Whole-mount fluorescence of the dorsal OB reveals GFP-expressing neurons innervating broadly distributed glomeruli. $\boldsymbol{A}$, Control mice expressing a Tet0-GFP transgene under the control of OMP-TTA illustrate the pattern of glomeruli in wild-type animals. $B-E$, OSNs expressing various OMP-tgORs target glomeruli that are broadly distributed throughout the dorsal $O B$ and similar in size to those of control mice (see insets for detail). The number of fluorescent glomeruli reflects the different levels of expression of the transgene. Generally $10-30 \%$ of OSNs express OMP-tgORs $(\boldsymbol{B}-\boldsymbol{D})$; however, OMP-MOR28 line $B(\boldsymbol{E})$ is expressed in $80-90 \%$ OSNs and OB fluorescence closely matches that of control mice $(\boldsymbol{A})$. $\boldsymbol{F}, \mathbf{G}$, Confocal images of the glomerular layer of OMP-tgOR mice counterstained with DAPI (blue) show that glomeruli targeted by OSNs expressing OMP-M72 (F) and OMP-MOR28 line B (G) appear filled with GFP- (and tgOR-) expressing fibers and are similar in morphology. Also note interspersed glomeruli not containing innervation from GFP-positive 0SNs in 0MP-M72 mice where the transgene is expressed in a subset (10-30\%) of OSNs $(\boldsymbol{F})$. Scale bars: $\boldsymbol{A}$ (for $\boldsymbol{A}-\boldsymbol{E}$ insets), $250 \mu \mathrm{m} ; \boldsymbol{D}$ (for $\boldsymbol{A}-\boldsymbol{E}), 1 \mathrm{~mm} ; \boldsymbol{F}$ (for $\boldsymbol{F}$ and $\boldsymbol{G}$ ), $50 \mu \mathrm{m}$. Medial (M) and anterior (A) directions in whole-mount images are indicated.

to more apical regions of the MOE. G $\gamma 8$ is primarily expressed in the basal, developing OSNs and is downregulated as neurons mature (Ryba and Tirindelli, 1995; Tirindelli and Ryba, 1996). In contrast, OMP is expressed in apical, fully mature OSNs (Rogers et al., 1987). There is some evidence that a small fraction of neurons expressing an OR are not fully mature (i.e., they do not contain OMP) (Iwema and Schwob, 2003), but precise information about when OR expression begins in a given OSN is lacking. Here we have investigated the effects of driving tgOR expression under the control of $\mathrm{G} \gamma 8$ ( $\mathrm{G} \gamma 8$-tgOR) on OSN targeting and the development of the olfactory system. Our results demonstrate that widespread expression of a $\mathrm{G} \gamma 8$-tgOR results in major anatomical and functional changes in the OB, implying that the timing of OR expression must be precisely controlled for normal olfactory development.

\section{Materials and Methods}

Genetically modified mice. The $\mathrm{rI7} \rightarrow \mathrm{M} 71$ mice (rI7 $\rightarrow$ M71-IRES-GFPIRES-taulacZ) carry a knock-in of the rat OR, rI7, in the M71 locus and thus express rI7 in the dorsal zone of the MOE; the OSNs expressing rI7 target a lateral glomerulus at the dorsal surface of the $\mathrm{OB}$ and coexpress both green fluorescent protein (GFP) and lacZ (Bozza et al., 2002). We also generated mice carrying a TetO-GFP-IRES-GFP transgene. All other strains used were as described previously (Nguyen et al., 2007). Mice were intercrossed to generate experimental animals as described below; the transgenes were hemizygous and knock-ins heterozygous.

Histology and fluorescence imaging. OB immunohistochemistry was performed on $40 \mu \mathrm{m}$ coronal floating sections (Marks et al., 2006). Pri- mary antibodies were rabbit anti- $\beta$-galactosidase (1:500; MP Biochemicals), rabbit anti- $\mathrm{G}_{\text {olf }}(1: 500$; Santa Cruz Biotechnology), and rabbit antityrosine hydroxylase and calbindin (1:2000; Millipore Bioscience Research Reagents). Floating sections were incubated with primary antibodies overnight at room temperature (for anti- $\beta$-galactosidase, antityrosine hydroxylase, and calbindin) or for $3 \mathrm{~d}$ at $4^{\circ} \mathrm{C}$ (for anti- $\mathrm{G}_{\text {olf }}$ ) and were detected using Cy3-conjugated secondary antibodies (1:1000; Jackson ImmunoResearch) for $2 \mathrm{~h}$ at room temperature. MOR2 8 immunohistochemistry was performed on $20 \mu \mathrm{m}$ coronal sections of the olfactory epithelium of 3-week-old OMP-TgMOR28 line B mice using a rabbit anti-MOR28 antibody (Barnea et al., 2004) (generously provided by Richard Axel, Columbia University, New York, NY) at 1:6000 dilution. Double-label in situ hybridization was performed on $10 \mu \mathrm{m}$ frozen sections using digoxigenin- and fluorescein-labeled antisense RNA probes at high stringency, as described previously (Hoon et al., 1999). Wholemount X-gal staining used standard methods (Mombaerts et al., 1996). Staining was performed at room temperature for $20 \mathrm{~h}$. Whole-mount images were collected on an Olympus SZX12 dissecting stereo microscope; regular photomicrographs were obtained using a Nikon Axiophot fluorescence microscope; and confocal microscopy ( $1 \mu \mathrm{m}$ optical sections) was with a Zeiss LSM-510-Meta (Carl Zeiss) or Leica TCS SP2 (Leica Microsystems). Images were processed using Adobe Photoshop.

Measurement of glomerular diameter. Images of coronal sections of the OBs from OMP-GFP, OMP-tgOR, G $\gamma 8$-tgOR, and G $\gamma 8+$ OMP-tgOR mice (at least three animals from each genotype) were measured using ImageJ (the diameter of glomeruli was defined as the longest axis). For G $\gamma 8$-tgOR mice, all glomeruli containing significant GFP were measured but glomeruli containing just a few GFP fibers (primarily in the ventral regions of the $\mathrm{OB}$ ) were ignored; $p$ values were calculated using a one-way 

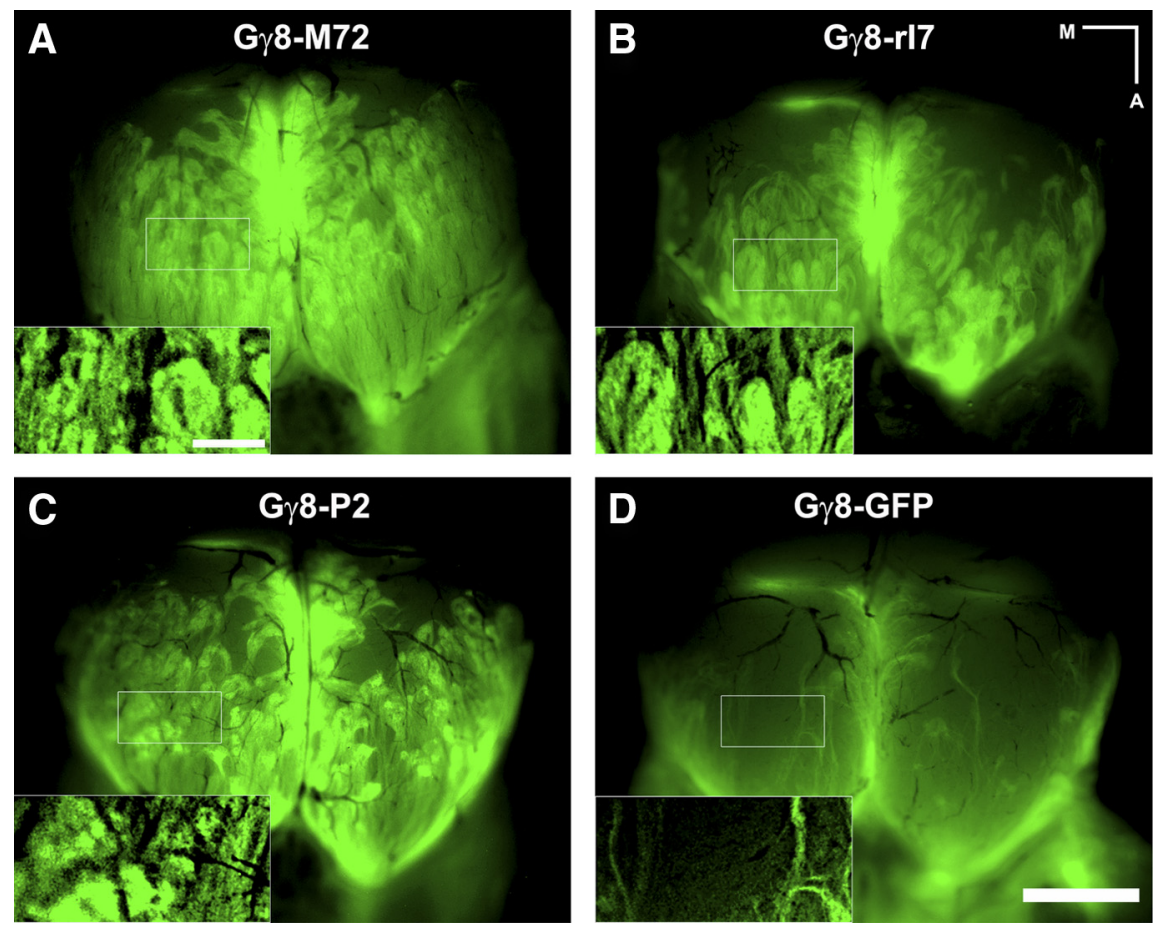

Figure 2. $\quad G \gamma 8$-tgOR OSNs innervate large and broadly distributed glomeruli. $\boldsymbol{A}-\boldsymbol{D}$, Whole-mount fluorescent images of the dorsal OB show projections of GFP-labeled OSNs expressing tgORs $(\boldsymbol{A}-\boldsymbol{C})$ or Tet0-GFP $(\boldsymbol{D})$ under the control of G $\gamma 8$-TTA. Note that OSNs expressing $\mathrm{G} \gamma 8$-tg0Rs innervate many large glomeruli ( $\boldsymbol{A}-\boldsymbol{C}$, insets), whereas $\mathrm{G} \gamma 8$-GFP-expressing 0SNs rarely enter the glomerular layer ( $\boldsymbol{D}$, inset). Scale bars: $\boldsymbol{D}$ (for $\boldsymbol{A}-\boldsymbol{D}), 1 \mathrm{~mm}$; insets, $250 \mu \mathrm{m}$. Medial $(M)$ and anterior $(A)$ directions are indicated.

ANOVA with Newman-Keuls posterior test to compare glomerular diameters of different mice.

Functional studies using intrinsic signal imaging. Mice were prepared and imaged using an Imager 3001 system (Optical Imaging) as described previously (Rubin and Katz, 1999; Belluscio and Katz, 2001). Approximately 5-week-old mice (at least six of each genotype) were used for measuring responses to ligands that activated tgOR. To denote regions of highest activity, images were imported into IPLab (Scanalytics). Pixels $>2$ SDs above the mean pixel value for that image were color coded and superimposed onto the blood vessel image. A mask was drawn to omit pixels located in peripheral bone and medial vessel regions not located over the dorsal bulbs. Animals that were at least 20 weeks old (seven or more mice for each odorant) were used to determine the distorted olfactory map to aliphatic ligands. All odorants used were of the highest purity available from Sigma and were dissolved at $1 \%$ in mineral oil.

\section{Results}

Mammalian odorant receptors have been shown to play an instructive role in targeting the axon of the OSNs in which they are expressed to appropriate locations in the olfactory bulb (Mombaerts et al., 1996; Wang et al., 1998), implying that their expression must occur before the primary neurons form permanent synapses with secondary olfactory neurons. We recently established a multicomponent, transgenic approach to express a single defined OR in a large proportion (up to $90 \%$ ) of OSNs by using an OSNdirected tetracycline-dependent transactivator to drive TetOcontrolled OR transgenes (Nguyen et al., 2007). In this system, the OSNs expressing tgORs are labeled by using an internal ribosomal entry site (IRES) to drive coexpression of GFP and the timing of OR expression is determined by the promoter used to drive TTA. In the present study, we used the OMP gene (Rogers et al., 1987), a marker of mature OSNs, to drive TTA at late stages of neural development and the $\mathrm{G} \gamma 8$ promoter (Ryba and Tirindelli, 1995 ) to target a more immature population of cells (Tirindelli and Ryba, 1996) and we examined the effects of tgOR expression on the architecture and function of the $\mathrm{OB}$.

When driven by an OMP-TTA knock-in allele, 36 of 37 lines of TetO-OR mice express a variety of different OMP-tgORs (e.g., OMP-M72, OMP-P2, OMP-rI7, OMPMOR28) in a large but incomplete subset (10-30\%) of OSNs (Nguyen et al., 2007). The remaining TetO-OR line (OMPMOR28 line B) consistently expressed the tgOR in many more neurons (up to $90 \%$ of OSNs) (Fig. 1 and supplemental Fig. 1, available at www.jneurosci.org as supplemental material). Independently, Fleischmann et al. (2008) developed a similar system and reported that when one particular tgOR, OMP-M71, is expressed in $\sim 90 \%$ of OSNs using the same OMPTTA driver, there is remarkably little disruption of the peripheral olfactory circuitry, the detection of odorant, or anatomical features of the olfactory bulb. Our results [Fig. 1, supplemental Fig. 1 (available at www.jneurosci.org as supplemental material), and data not shown] substantiate some of these conclusions, showing that expressing a variety of different tgORs late in OSN development has very little effect on the organization of the olfactory system. In all cases, the neurons expressing the transgene project to widely scattered glomeruli throughout the olfactory bulb (Fig. 1 and supplemental Fig. 1, available at www.jneurosci.org as supplemental material) that are structurally indistinguishable from glomeruli in control animals where the OMP-TTA knock-in drives expression of TetO-GFP alone.

\section{Targeting and function of OSNs expressing G $\gamma 8$-tgORs}

We have also generated many lines of $\mathrm{G} \gamma 8$-tgOR mice wherein a G $\gamma 8$-TTA transgene drives expression of the TetO-ORs (Nguyen et al., 2007). G $\gamma 8$ is primarily expressed in immature OSNs with cell bodies localized to more basal regions of the MOE than those expressing OMP, and range in appearance from unstructured cells to typical OSN morphology (Tirindelli and Ryba, 1996). In 3-week-old animals, a large fraction of OSNs still express G $\gamma 8$ tgORs, but in older mice, expression is downregulated as neural proliferation decreases (Ryba and Tirindelli, 1995; Nguyen et al., 2007). Whole-mount images of the dorsal OB of 3 -week-old G $\gamma 8$-tgOR mice reveal widespread projections of GFP-labeled, tgOR-expressing fibers that innervate many abnormally large glomeruli (Fig. $2 A-C$ ). In contrast, OSNs expressing a TetO-GFP transgene under the control of the $\mathrm{G} \gamma 8$-promoter (G $\gamma 8$-GFP) label the nerve layer but very few fibers enter the glomerular layer (Fig. 2D). We hypothesized that expression of a tgOR in an immature neuron might alter the normal course of neural development and persist even after $\mathrm{G} \gamma 8$ expression would be downregulated in mice not expressing a tgOR. Indeed, all 3-week-old G 88 -tgOR mice exhibited a thicker layer of GFP-expressing OSNs (Nguyen et al., 2007) than expected from the expression of G $\gamma 8$ (Ryba and Tirindelli, 1995; Tirindelli and Ryba, 1996). Remarkably, two color double-label in situ hybridization studies demonstrated that expression of $\mathrm{G} \gamma 8$-tgORs is not limited to basal cells but is also observed in more apical OSNs in which 


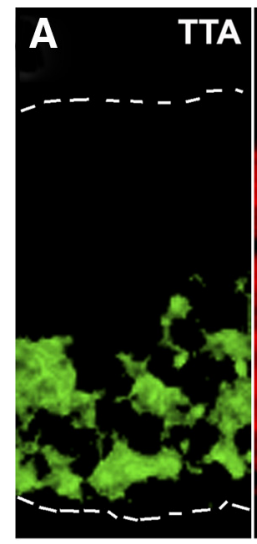

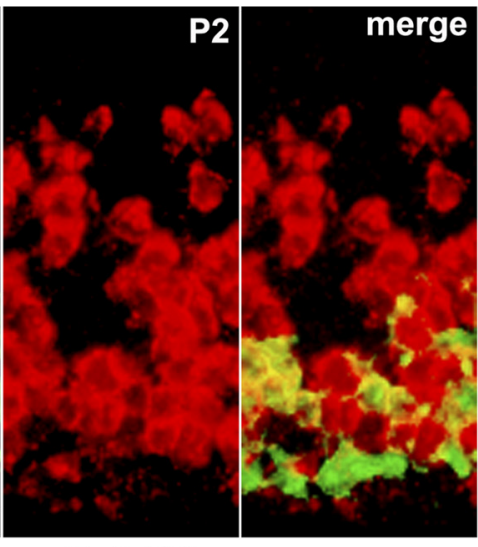

G $\gamma \mathbf{8 - P 2}$
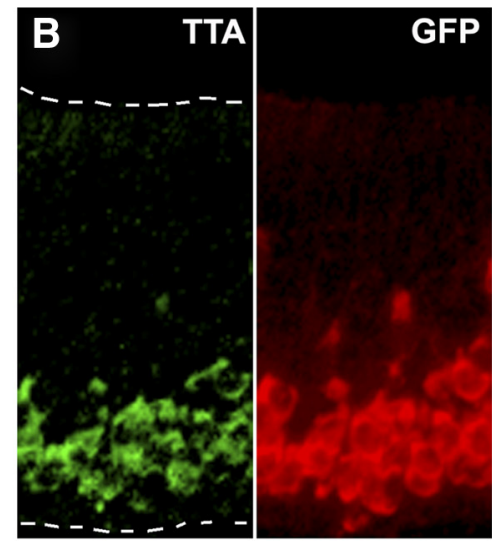

G $\gamma 8$-GFP

Figure 3. Expression of $\mathrm{G} \gamma 8$-tgOR continues even after downregulation of $\mathrm{G} \gamma 8$-TTA. Double-label in situ hybridization was used to analyze expression of Tet0-P2 (A, red) or Tet0-GFP ( $\boldsymbol{B}$, red) and $G \gamma 8$-TTA $(A, B$, green) in the MOE of 3-week-old mice. The expression of $G \gamma 8-P 2(A$, red) illustrates that tgORs are expressed in OSNs containing TTA but, importantly, persists in many more mature (apical) neurons where $\mathrm{G} \gamma 8$-TTA is no longer detectable. In contrast, $\mathrm{G} \gamma 8$-GFP expression ( $\boldsymbol{B}$, red) closely matches that of $\mathrm{G} \gamma 8$-TTA; the basal and apical extent of the epithelium are indicated by dotted lines in the left panels of $A$ and $B$. Scale bar, $20 \mu \mathrm{m}$.

G $\gamma 8$-TTA is no longer detectable (Fig. $3 A)$. By comparison, G $\gamma 8$-GFP expression always matches that of TTA (Fig. $3 B$ ). In this regard, it is also notable that a few cells at the base of the epithelium express TTA but not the tgOR (Fig. $3 A$ and data not shown); this discrepancy is never seen with G $\gamma 8$-GFP (Fig. $3 B$ ) but is common to all $\mathrm{G} \gamma 8$-tgOR lines.

When G $\gamma 8$-TTA and OMP-TTA are used in combination to drive TetO-OR transgenes ( $\mathrm{G} \gamma 8+\mathrm{OMP}$-tgOR mice), most OSNs express a tgOR (Nguyen et al., 2007). These mice provide an important control to establish whether the pattern of glomerular innervations of $\mathrm{G} \gamma 8$-tgOR OSNs is transient. In fact, Figure $4 A$ demonstrates that GFP fluorescence in the $\mathrm{OB}$ of 3-week-old G $\gamma 8+$ OMP-tgOR mice closely resembles that of $\mathrm{G} \gamma 8$-tgOR mice. Moreover, in 6-month-old G $\gamma 8+$ OMP-tgOR animals, this pattern of GFP fluorescence is preserved with innervation of many large glomeruli (Fig. $4 \mathrm{~B}$ and see supplemental Fig. 2, available at www.jneurosci.org as supplemental material, for the distribution and size of GFP-positive glomeruli throughout the bulb). Importantly, the size of GFP-labeled glomeruli always appeared to be significantly larger in mice containing a $\mathrm{G} \gamma 8$-tgOR transgene than in control animals or mice expressing OMP-tgORs (Figs. 1, 2, and 4). Therefore we examined sections through the olfactory bulb of the four groups of mice to more accurately assess the influence of expressing $\mathrm{G} \gamma 8$-tgORs. Figure 5 shows examples of the morphology of glomeruli in OMP-GFP, OMP-tgOR, G $\gamma 8$-tgOR, and G $\gamma 8+$ OMP-tgOR mice together with quantitative analysis of the GFP-labeled glomeruli. In G $\gamma 8$-tgOR and G $\gamma 8+\mathrm{OMP}$-tgOR, but not OMP-tgOR, animals, the GFP-labeled glomeruli are morphologically distinct from those of OMP-GFP controls, including some that are dramatically enlarged (Fig. 5). Indeed, the mean diameter of fluorescent glomeruli in G $\gamma 8$-tgOR and $\mathrm{G} \gamma 8+\mathrm{OMP}-\operatorname{tgOR}$ mice was $\sim 1.4$ times that of control or OMP-tgOR mice, equating to a 2.5-fold to threefold increase in average glomerular volume for OSNs expressing a $\mathrm{G} \gamma 8$-tgOR.
Interestingly, $\mathrm{G}_{\text {olf }} \alpha$-staining revealed smaller glomeruli that did not contain GFP fibers interspersed among their much larger fluorescent counterparts in the dorsal OB of $\mathrm{G} \gamma 8$-tgOR animals (supplemental Fig. 3, available at www.jneurosci.org as supplemental material). As noted previously, $50-70 \%$ of OSNs express transgenic receptors in 3-week-old G $\gamma 8$-tgOR mice (Nguyen et al., 2007). Therefore, a significant number of neurons express ntORs in the Gy8-tgOR animals and likely target these smaller glomeruli that are largely devoid of GFP and tgOR-OSN innervation. Sections through the OB of 3-week-old G $\gamma 8$-tgOR mice stained for OSN markers revealed an approximately normal glomerular count. Because the size of the bulbs of transgenic mice was similar to that of control animals, this likely reflects the decrease in size of ntORglomeruli that offsets the increased size of tgOR-glomeruli.

We have previously shown that two G $\gamma 8$-tgORs, G $\gamma 8$-rI7 and G $88-\mathrm{M} 72$, selectively activate OSNs in the MOE in response to $n$-octanal and acetophenone, respectively (Nguyen et al., 2007). Therefore, we investigated bulbar activity induced by activation of the olfactory epithelium through these G $\gamma 8$-tgORs. Intrinsic signal imaging (ISI) provides an effective approach to study functional responses in the $\mathrm{OB}$ based on activity-dependent changes in blood flow and oxygenation state resulting from localized neural activity (Rubin and Katz, 1999; Belluscio and Katz, 2001). As shown in Figure 6, young G $\gamma 8$-M72 or G $\gamma 8$-rI7 mice responded with robust ISI signals to ligands delivered to the MOE. The 


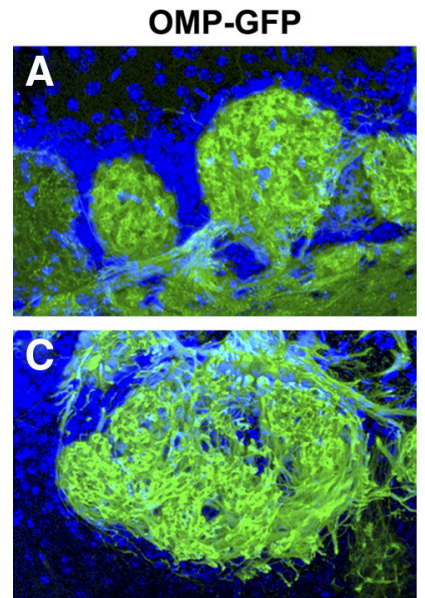

G $\gamma 8-$ rl7
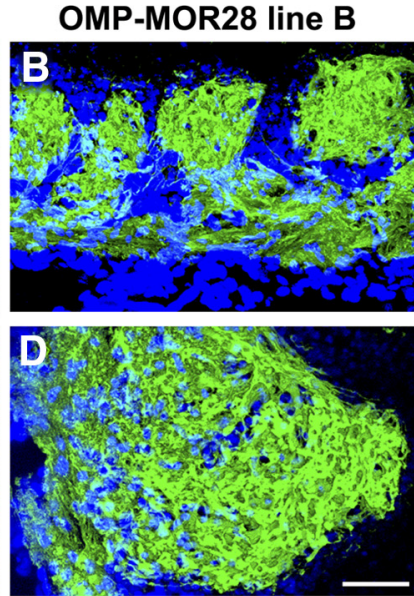

G 8 8+OMP- rl7
E

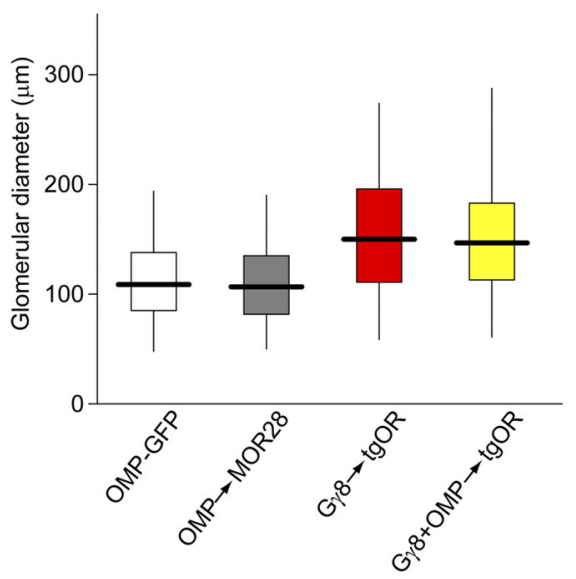

Figure 5. Qualitative and quantitative analysis of glomerular size in tgOR mice. $A-D$, Confocal images of the dorsal OB of mice expressing OMP-GFP $(\boldsymbol{A})$ or tgORs under the control of OMP $(\boldsymbol{B}), \mathrm{G} \gamma 8(\boldsymbol{C})$, or both $\mathrm{G} \gamma 8+0 \mathrm{MP}$ promoters $(\boldsymbol{D})$ show transgene expression (green) and are counterstained with DAPI (blue). C, D, In animals carrying a $G \gamma 8$-TTA transgene, some of the labeled glomeruli are substantially larger than the largest glomeruli of control animals. Scale bar, $50 \mu \mathrm{m}$. E, Photomicrographs of 3-week-old animals were used for quantitative analysis of GFP-labeled glomerular diameter for OMP-GFP controls ( $n=553$ glomeruli), OMP-tg0R (MOR28 line B, $n=414$ ), G $\gamma 8$-tg0R (M72 and rl7, $n=277$ ), and G $\gamma 8+0 M P-\operatorname{tg} 0 R$ (M72 and $\mathrm{rl} 7, n=434$ ). The plot indicates the median glomerular diameter (solid line), the 25 th and 75 th percentiles (boxed) and the 2.5 and 97.5 percentiles (whiskers); the mean glomerular diameter of $\mathrm{G} \gamma 8$-tgOR and $\mathrm{G} \gamma 8+0 \mathrm{MP}$-tgOR mice is significantly larger than that of control or OMP-tgOR animals ( $p<0.0001$ in each case). Glomerular diameter increased in size regardless of direction of sections, thus the presence of a $G \gamma 8$-tgOR results in an approximately threefold increase in volume.

responses closely matched the projection pattern of OSNs expressing tgORs and, importantly, were highly selective for the appropriate ligand; G $\gamma 8$-M72 mice responded strongly to acetophenone but not octanal, whereas G $\gamma 8$-rI7 animals showed the opposite sensitivity.

\section{Modified targeting of the OSNs expressing nontransgenic ORs}

To study the targeting of ntOR OSNs, we used two lines of mice in which OSNs expressing specific ntORs were marked by coexpression of LacZ (Mombaerts et al., 1996; Bozza et al., 2002). In one, the ntOR is the rat $\mathrm{I} 7$ receptor knocked into the M71 locus (rI7 $\rightarrow$ M71-lacZ, see Materials and Methods for details); the neurons expressing this ntOR are localized to the dorsal MOE where G $\gamma 8$-tgOR expression is highest (Nguyen et al., 2007). Figure 7 demonstrates that 3-week-old mice carrying both the rI7 $\rightarrow$ M71 gene and G 88 -tgOR have very few LacZ-positive neurons that project to scattered regions of the dorsal olfactory bulb rather than to the typical site of the $\mathrm{rI7} \rightarrow \mathrm{M} 71$ glomerulus (Bozza et al., 2002). In the other ntOR-LacZ line, the labeled ntOR is P2-lacZ, which is more ventrally expressed. By counting LacZ-stained OSNs, we determined that a $\mathrm{G} \gamma 8$-tgOR background resulted in approximately a twofold decrease in number of $\mathrm{P} 2$ neurons; the number of lacZ-positive cells on the third turbinate decreased from $180.9 \pm 5.8$ in 3-week-old control mice $(n=25$, mean \pm SEM) to $96.8 \pm 6.1$ in G $\gamma 8$-tgOR animals $(n=30)$. Interestingly, LacZ staining revealed that the majority of P2 neurons still project to glomeruli in the appropriate medial and lateral parts of the OB (Mombaerts et al., 1996) of these animals, but a significant subset of P2 OSNs are also frequently mistargeted to other regions of the $\mathrm{OB}$ (Fig. 7D-F). Approximately 60\% (63 of 101 OBs) of 3-week-old G $\gamma 8$-tgOR animals exhibited mistargeting of a substantial fraction of $\mathrm{P} 2$ neurons similar to that illustrated in Figure 7. These mistargeted OSNs often form small, ectopic P2 glomeruli (Fig. $7 J$ ) or enter glomeruli of all sizes that contain projections from OSNs expressing tgORs (Fig. $7 \mathrm{H}, \mathrm{I}$ ).

Does expressing a tgOR differentially affect the projection patterns of the OSNs expressing the identical ntOR? To answer this question, we generated mice carrying a labeled endogenous allele, P2-lacZ, and a G $\gamma 8$-P2 transgenic receptor (Fig. 8). Surprisingly, we found that targeting of the native receptor $\mathrm{P} 2-$ lac $Z$ appeared no different in $\mathrm{G} \gamma 8$-P2 and other G $\gamma 8$-tgOR lines (compare Figs. $7 D-F$ and $8 A$ ). Similarly, the few remaining projection of $\mathrm{rI7} \rightarrow$ M71-lacZ OSNs exhibited indistinguishable patterns of projection whether they were in the identical ( $\mathrm{G} \gamma 8$-rI7) or a distinct (G $\gamma 8-\mathrm{M} 72)$ transgenic background (Fig. $7 \mathrm{~B}, \mathrm{C}$ ).

$\mathrm{G} \gamma 8$ expression declines dramatically once the rapid growth phase of the MOE ends at $~ 3$ weeks (Ryba and Tirindelli, 1995). Therefore, we investigated whether the effects of G $y 8$-tgOR expression on mistargeting of ntOR-OSNs change as the animals age. Supplemental Figure 4 (available at www.jneurosci.org as supplemental material) demonstrates that, in older adult animals, G $\gamma 8$-tgORs are restricted to regenerating neurons at the base of the MOE. In these mice, tgOR-expressing OSNs are still visible in the outer nerve layer of the $\mathrm{OB}$ but few appear to enter glomeruli. Nonetheless, P2-lacZ-expressing OSNs continue to exhibit the same degree of mistargeting (47 of 62 OBs of mice $\geq 20$ weeks old) as observed in younger animals, regardless of the nature of the G $\gamma 8$-tgOR (compare Figs. 7 and 8).

\section{Persistent functional changes induced by $\mathrm{G} \gamma 8$-mediated tgOR expression}

Since older $\mathrm{G} \gamma 8$-tgOR mice prominently express ntORs in the MOE, we hypothesized that ISI would provide a useful measure of their functional olfactory responses and the consequences of the distorted map of ntOR-OSN connections. Aliphatic odorants are known to activate multiple glomeruli in the dorsal $\mathrm{OB}$ of wild-type mice (Belluscio and Katz, 2001) and also produced strong ISI signals in mature adult G $\gamma 8$-tgOR animals (Fig. 9). However, the pattern of activation revealed by ISI was grossly distorted in G $\gamma 8$-tgOR mice relative to control animals; individual glomeruli were no longer distinguished and there was a marked rostral shift in ISI signal for all odorants tested (Fig. 9). Indeed, whereas ethyl $n$-butyrate, $n$-propanal, and $n$-butanal induce readily distinguishable patterns of glomerular activity in control mice, they all activate a broad overlapping region of the rostral $\mathrm{OB}$ in $\mathrm{G} \gamma 8$-tgOR animals. 


\section{Discussion}

Widespread expression of a single transgenic OR late in the development of OSNs has few anatomical, functional, or developmental consequences (Fig. 1) (Fleischmann et al., 2008). In contrast, we now show that early expression of a $\mathrm{G} \gamma 8$-tgOR has profound effects on the wiring and anatomy of the peripheral olfactory system at multiple levels. First, whereas few G $\gamma 8$ expressing neurons normally enter the glomerular layer, many neurons expressing $\mathrm{G} \gamma 8$-tgORs target prominent glomeruli. At least in part this is because tgOR expression persists in OSNs even after expression of $\mathrm{G} \gamma 8$ (and G $\gamma 8$-TTA) is downregulated. Second, although the OSNs expressing G $\gamma 8$-tgORs do not cluster to form a single massive glomerulus, these neurons innervate glomeruli that are approximately three times larger in volume than those of wild-type mice. Third, G $\gamma 8$ tgOR expression leads to disruption of one of the most invariant features of the peripheral olfactory system: OSNs expressing a particular OR project to fixed locations in the OB. Notably, mice with knock-out of essential signaling molecules (Belluscio et al., 1998; Lin et al., 2000) or even lacking the OB-projection neurons and interneurons (Bulfone et al., 1998) display appropriate OSN targeting in the OB. However, precocious expression of a tgOR under the control of the G $\gamma 8$-promoter disrupts the normal targeting of OSNs, leading to permanent changes in the functional map of olfactory responses in the $\mathrm{OB}$.

\section{Targeting and development of Gr8-tgOR-expressing OSNs}

OSNs of mice expressing an OMP-tgOR project axons throughout the $\mathrm{OB}$, producing separate and distinct glomeruli (Fig. 1). These glomeruli are normally sparse but are relatively evenly distributed throughout the $\mathrm{OB}$ and closely resemble those of control animals in size and shape,

indicating that glomerular structure might be tightly regulated as suggested previously (Fleischmann et al., 2008). In contrast, expression of G $\gamma 8$-tgORs significantly increases the size of the glomeruli innervated by transgene-expressing OSNs (Fig. 5). Nonetheless, the size of glomeruli still appears to be constrained, implying that other factors also play a role in determining glomerular boundaries. Given that very few labeled OSNs ever project beyond the glomerular layer into the exterior plexiform layer of the OB (supplemental Fig. 2, available at www.jneurosci. org as supplemental material), we hypothesize that attractive or repellant cues, in addition to ORs, must limit at least one dimension of glomeruli. Why are $\mathrm{G} \gamma 8$-tgOR glomeruli larger than those in control or OMP-tgOR mice? Interestingly, during early development of the olfactory system, OSNs expressing a defined recep-

\section{acetophenone}
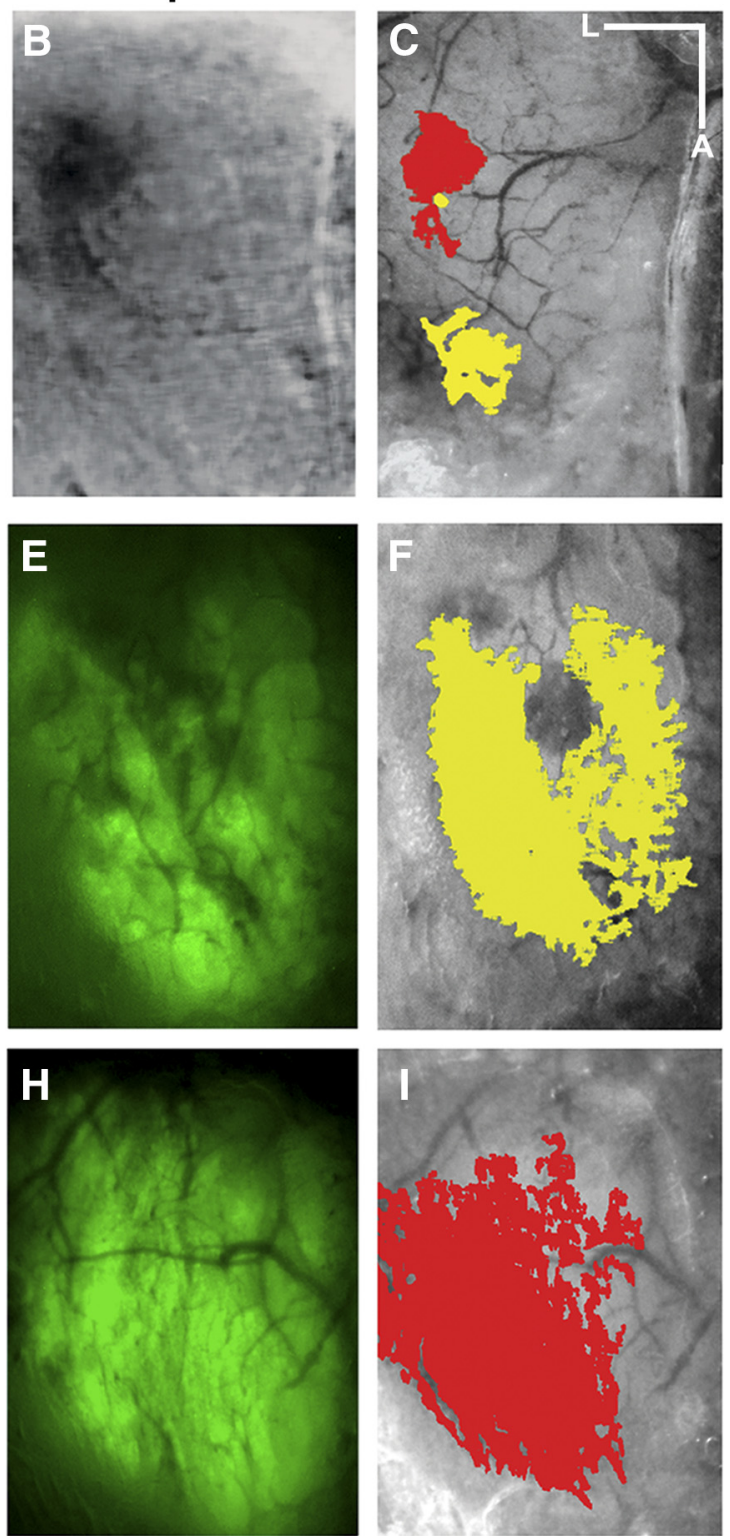

Figure 6. Activation of tgORs by odorants leads to widespread activity in the $\mathrm{OB}$. $\mathbf{A}-\mathbf{C}$, In control animals, ISI reveals discrete regions of activity (dark spots) elicited by octanal $(\boldsymbol{A})$ and acetophenone $(\boldsymbol{B})$; peak areas of activity have been color coded (octanal, cognate ligands, $G \gamma 8$-rl7 to octanal $(\boldsymbol{D}, \boldsymbol{F})$ and $\mathrm{G} \gamma 8 \mathrm{-M} \mathrm{M} 72$ to acetophenone $(\boldsymbol{G}, \boldsymbol{I})$, that correspond with transgene expression (GFP fluorescence, $\boldsymbol{E}, \boldsymbol{H})$. Scale bar, $250 \mu \mathrm{m}$. Lateral (L) and anterior (A) directions are indicated.

tor first project to the appropriate region of the OB but spread diffusely over a relatively large area (a protoglomerulus) that later condenses to form a typical glomerulus (Potter et al., 2001). Not only are the $\mathrm{G} \gamma 8$-tgOR glomeruli larger than normal glomeruli but they also appear more diffuse (Fig. 2). However, unlike protoglomeruli, they never condense. Figure 4 demonstrates that G $88+$ OMP-tgOR glomeruli maintain this enlarged and diffuse morphology even when OMP-TTA is responsible for almost all tgOR expression. We speculate that precocious OR expression in immature OSNs might accelerate their innervation of the OB, allowing them to accumulate in the glomerular layer with reduced competition from OSNs expressing other receptors and thus give rise to the observed phenotype of enlarged glomeruli.

Intrinsic signal imaging demonstrated that cognate ligands induce robust changes in hemoglobin oxygenation in G $\gamma 8-\mathrm{M} 72$ 

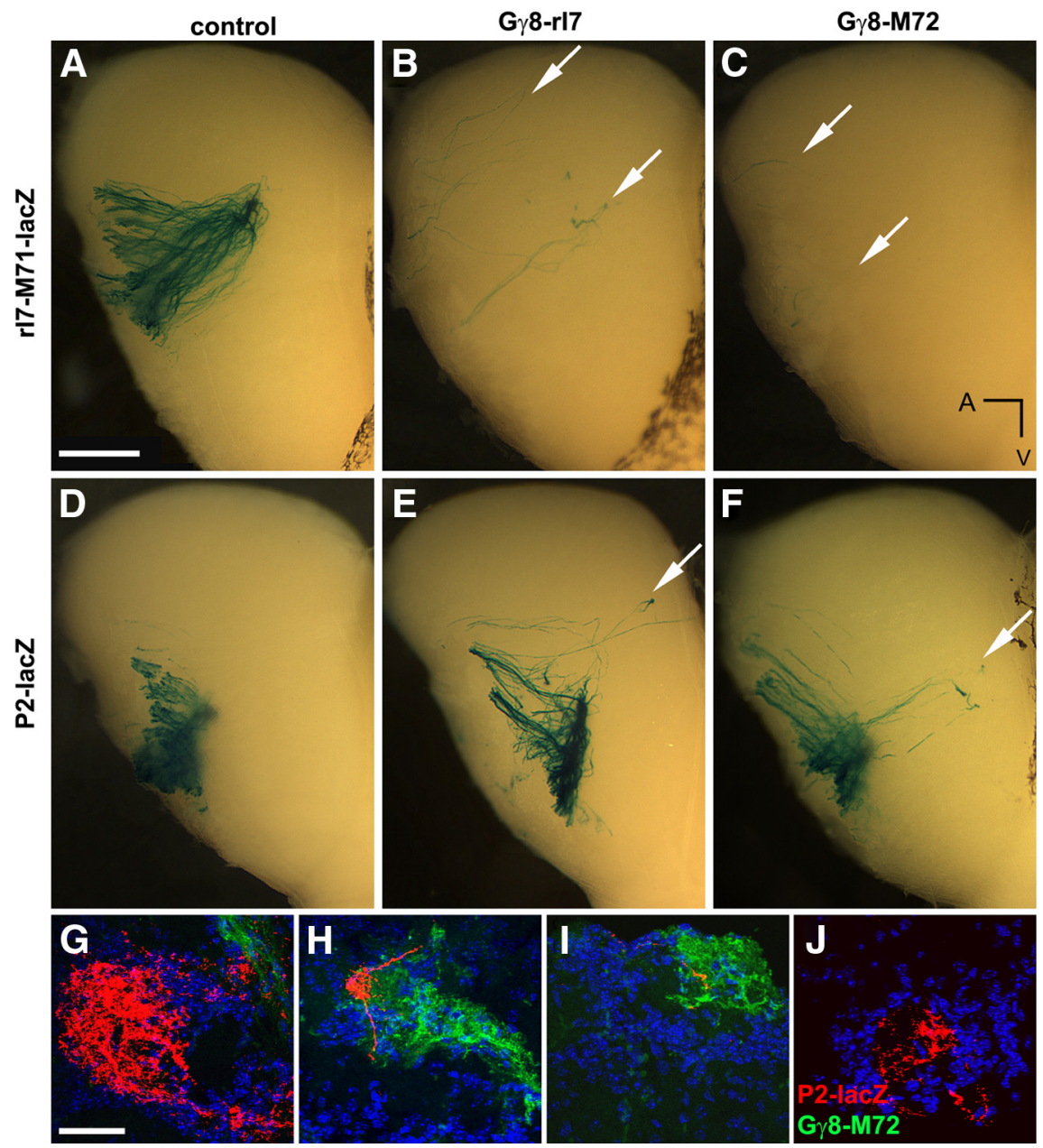

Figure 7. G G8-tgORs disrupt targeting of OSNs expressing endogenous receptors. $\boldsymbol{A}-\boldsymbol{F}$, Medial view of the $0 \mathrm{~B}$ from 3-week-old heterozygous rl7 $\rightarrow$ M71-lacZ (A-C) and P2-lacZ (D-F) mice stained for LacZ. In control rl7 $\rightarrow$ M71-lacZ mice $(\boldsymbol{A})$, hundreds of labeled axons project to the expected location in the $O B$; in contrast, mice expressing $G$ y8-rl7 (B) or G y8-M72 ( $C$ have many fewer stained fibers that meander without convergence (arrows). Less dramatic mistargeting was detected in P2-LacZ mice $(\boldsymbol{D}-\boldsymbol{F})$. In control animals, P2-lacZ OSNs target a single glomerulus in the medial OB (D). In G $\gamma 8$-rl7 (E) or Gy8-M72 (F) mice, the majority of P2-LacZ-stained fibers innervate a glomerulus in the appropriate region of the $\mathrm{OB}$ but a subset of P2-OSNs typically project to distal regions of the $0 B$ and form ectopic glomeruli (arrows). $\mathbf{G}-J$, Confocal images of the $0 B$ from $G \gamma 8-M 72$ mice immunostained for LacZ expression (red), counterstained with DAPI (blue), and examined for tgOR expression (green, GFP). The major P2 glomeruli rarely contain any innervation from $\mathrm{tg} O \mathrm{R}$ OSNs, as indicated for a medial $\mathrm{P} 2 \mathrm{~g}$ lomerulus (G). Ectopic $\mathrm{P} 2$ neuron projections focus on glomeruli that also contain tgOR-OSN innervation $(\boldsymbol{H}, \boldsymbol{I})$ as well as regions of small glomeruli that do not contain tgOR expressing axons (J). Scale bars: (in $\boldsymbol{A}) \boldsymbol{A}-\boldsymbol{F}, 500 \mu \mathrm{m}$; (in $\mathbf{G}) \mathbf{G}-\boldsymbol{J}, 50 \mu \mathrm{m}$. Ventral (V) and anterior (A) directions in whole-mount images are indicated.

and G $\gamma 8$-rI7 mice (Fig. 6). Thus, it appears that wide regions of the $\mathrm{OB}$ of $\mathrm{G} \gamma 8$-tgOR mice are activated by ligands that stimulate the transgene. In contrast, the most striking effect of expressing OMP-M71 in mature OSNs was that the mice failed to respond to the cognate ligand acetophenone (Fleischmann et al., 2008). This insensitivity to the ligand was not at the level of reception but instead was largely attributed to the action of inhibitory circuits, e.g., between glomeruli in the OB (Fleischmann et al., 2008). Interestingly similar inhibitory interactions have been observed in the Drosophila antennal lobe (Olsen and Wilson, 2008), suggesting that mice and flies may have converged on the same solution to extract salient olfactory information from complex mixtures. The distribution of major classes of inhibitory interneurons in the glomerular layer of all types of tgOR mice is similar to that of control animals (supplemental Fig. 5, available at www.jneurosci.org as supplemental material). Therefore, we hypothesize that differences in the completeness of OR expression [ $50-70 \%$ for G $\gamma 8$-tgOR mice vs $95 \%$ of OSNs in OMP-M71 animals (Fleischmann et al., 2008)] and the presence of interspersed glomeruli that do not have significant tgOR-OSN innervations (supplemental Fig. 3, available at www. jneurosci.org as supplemental material) most likely reduce the degree of inhibition and thus allow propagation of responses mediated by $\mathrm{G} \gamma 8$-tgORs.

$\mathrm{G} \gamma 8$ is expressed in immature OSNs, most of which neither express OMP nor innervate glomeruli. Similarly, G $\gamma 8$-TTA and G $\gamma 8$-TTA-driven TetO-GFP are primarily found in OSNs that do not extend axons into glomeruli. In contrast, G $\gamma 8$ tgOR-OSNs clearly innervate many glomeruli (Fig. 2) and activation of Gy8-tgORs leads to widespread activity in the OB (Fig. 6 ). We noted that Gy8-tgOR expression in the MOE extended into the OMPpositive cell layer for all Gy8-tgOR lines (Nguyen et al., 2007) and, remarkably, that the OMP/tgOR-positive cells do not express detectable TTA mRNA (Fig. 3). Feeding mice doxycycline to block TTAdependent transcription abolished tgOR expression (data not shown), demonstrating that all transgene expression relies on the TTA transcription factor. Given that no extended expression zone was detected for other TetO-transgenes, including GFP (Fig. 3 ) as well as a taste receptor, mT2R16, and the $\kappa$-opioid receptor RASSL (data not shown), it appears that the tgOR is crucial for the prolonged expression of the transgene. One attractive possibility is that the OR coding sequence stabilizes the association of TTA with the TetO repeats, perhaps even by recruiting factors that are normally involved in OR gene regulation, to form a larger transcription complex. In addition, we observed delayed expression of many different G $\gamma 8$-tgORs, e.g., as typified by TTA-positive but tgOR-negative cells at the base of the epithelium (Fig. $3 A$ ). Most probably this also reflects the complex nature of OR gene regulation (Nguyen et al., 2007).

\section{Effects of widespread expression of transgenic ORs on OSNs not expressing the transgene}

Axons of OSNs expressing the same OR normally target glomeruli that are foci for that particular receptor and do not contain axons from OSNs expressing other ORs. Expression of an OMPtgOR transgene does not appear to disrupt the focused projection of OSNs expressing ntORs (Fleischmann et al., 2008). One caveat is that in lines with extensive transgene expression like OMPMOR28 line B (Fig. 1 and supplemental Fig. 1, available at www. jneurosci.org as supplemental material), ntORs may project to the expected location but cannot target normal homotypic glomeruli as the glomerular layer of such animals is filled with GFPpositive fibers. Notably, in other OMP-tgOR lines, GFP-labeled glomeruli are also largely (or exclusively) composed of OSNs expressing the transgene despite its expression in a relatively lim- 

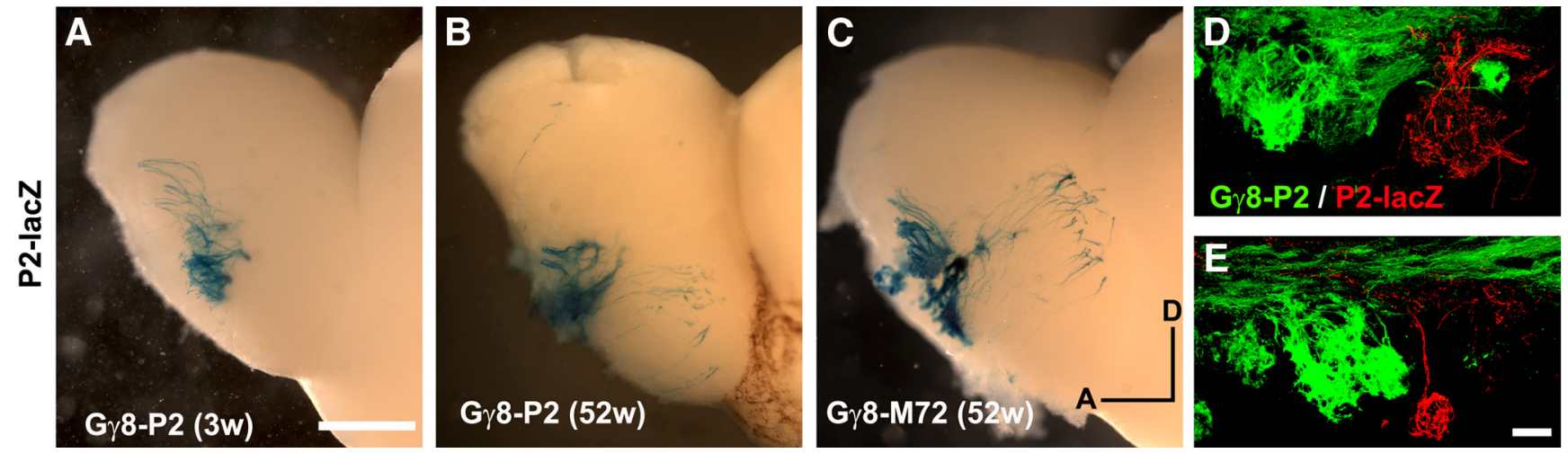

Figure 8. Projections of $\mathrm{P} 2$-lacZ OSNs in mice expressing a $\mathrm{G} \gamma 8$ - $\mathrm{P} 2$ transgene reveal that transient, precocious $\mathrm{G} \gamma 8$ - $\mathrm{tg}$ OR expression (not the identity of the tgOR) affects targeting of nt0R-OSNs. $A-C$, Representative whole-mount images of lacZ staining in the medial $0 B$ of P2-lacZ mice expressing $\mathrm{G} \gamma 8$-tg0Rs show that although most neurons project to a glomerulus in the appropriate region of the $0 B$, some fibers target ectopic regions of the bulb both in young (3-weeks-old, $A$ ) and old (52-weeks-old, $B, C$ ) animals. Notably, G $\gamma 8$-tgOR expression is restricted to a very small subset of neurons in these older animals (supplemental Fig. 4, available at www.jneurosci.org as supplemental material). These data also reveal that a $\mathrm{G} \gamma 8$ - $\mathrm{P} 2$ transgene has a similar effect on the targeting of ntOR-0SNs expressing the very same OR from the endogenous locus as any other $\mathrm{G} \gamma 8$-tgOR. Equivalent extents of P2-lacZ mistargeting were apparent in 52-week-old G $\gamma 8$-P2 (B) and $\mathrm{G} \gamma 8$-M72 mice ( $\boldsymbol{C}$, as well as in young animals (compare $\boldsymbol{A}$ with Fig. 7). Confocal images of P2-lacZ-positive (immunofluorescence, red) and $\mathrm{G} \gamma 8$-P2-expressing fibers (GFP, green) highlight the segregated projections of endogenously and transgenically encoded P2-expressing OSNs to the medial (D) and lateral (E) P2 glomeruli. Scale bars: (in $\boldsymbol{A}$ ) $\boldsymbol{A}-\boldsymbol{C}, 500 \mu \mathrm{m}$; (in $\boldsymbol{E}) \boldsymbol{D}, \boldsymbol{E}, 50 \mu \mathrm{m}$. Dorsal (D) and anterior (A) directions in whole-mount images are indicated.

ited subset of OSNs (Fig. 1). Thus, even though OMP is a marker of mature OSNs, OMP-driven tgORs appear to be able to influence some aspects of axonal projection.

G 88 -tgOR mice also exhibit extensive (but less complete) convergent projections of both tgOR- and ntOR-expressing OSNs. Neurons expressing tgORs generally innervate large glomeruli whereas the majority of neurons that express one particular ntOR, P2-lacZ, project to glomeruli that do not contain tgOR fibers. Remarkably, the extensive segregation of tgOR-and ntOR-expressing OSNs is maintained even when the receptor in both sets of labeled neurons is identical (i.e., in mice expressing P2-lacZ and Gy8-P2). Thus, homotypic clustering of neurons expressing the same receptor cannot be simply mediated by the OR identity and may involve other aspects of its expression, e.g., level at a particular developmental stage is likely a second factor (Feinstein and Mombaerts, 2004; Feinstein et al., 2004; Zou et al., 2004). We observed significant mistargeting of ntOR-expressing OSNs in G $\gamma 8$ tgOR mice. For example, P2-lacZ ntOSNs target ectopic glomeruli, some of which contain tgOR fibers (Fig. 7). Notably, nontransgenic OR mistargeting is particularly prominent in the dorsal $\mathrm{OB}$ where $\mathrm{G} \gamma 8$-tgOR is highest. Here we detected random projections of the few remaining $\mathrm{rI7} \rightarrow$ M71-lacZ labeled neurons (Fig. 7) and extensive distortion of the functional map of odorant responses (Fig. 9). We envision at least three distinct scenarios that together may help explain our observations. First, the decreased number of OSNs expressing a particular ntOR might preclude formation of stable directions are indicated. control
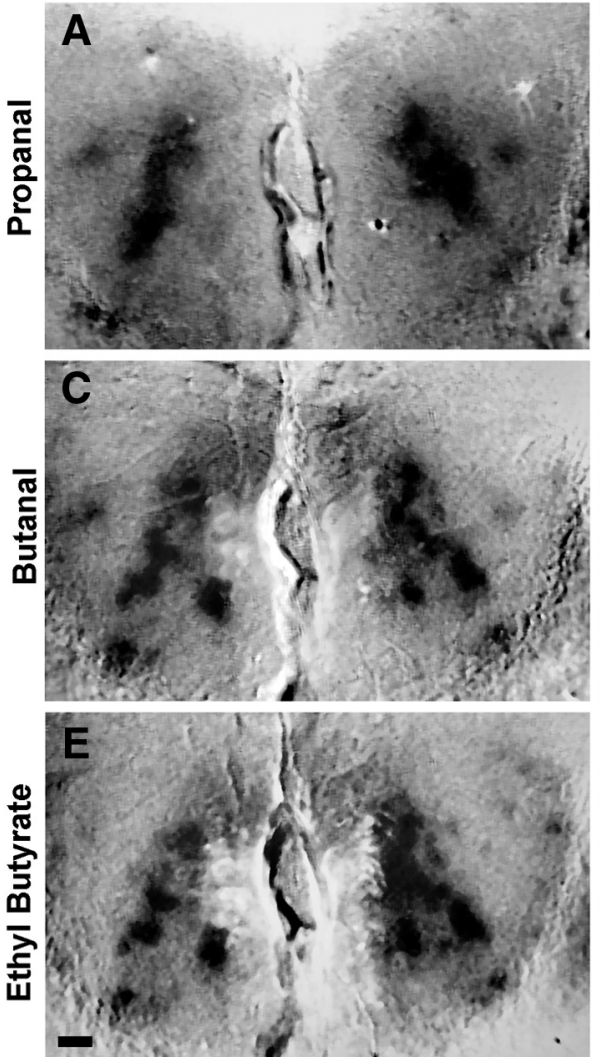

G $\gamma 8-\mathbf{M 7 2}$
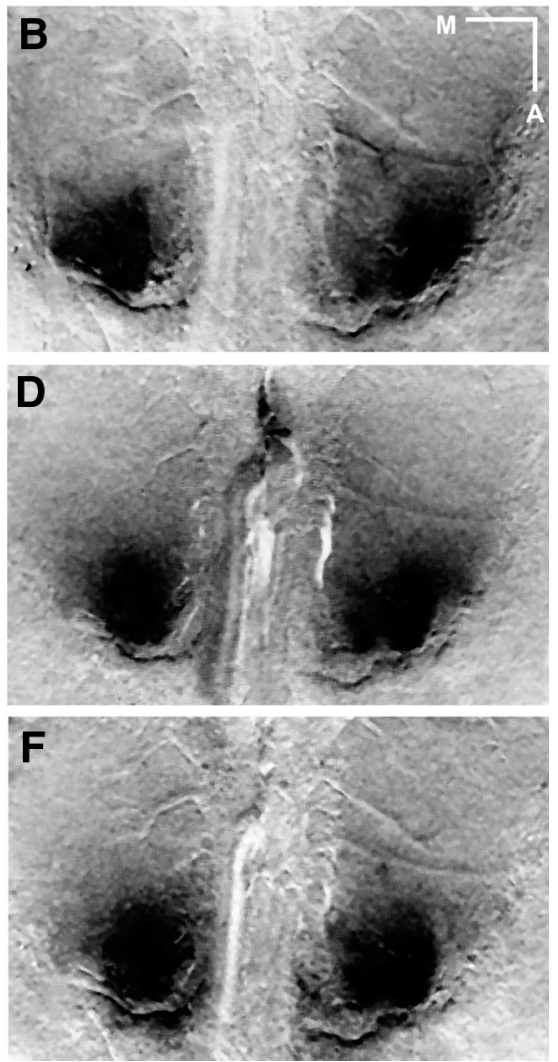

Figure 9. G $\quad G 8$-tgOR expression dramatically changes functional activity in the dorsal $O B$. $A-F, I S I$ illustrating $O B$ activity elicited by $n$-propanal $(\boldsymbol{A}, \boldsymbol{B}), n$-butanal $(\boldsymbol{C}, \boldsymbol{D})$, and ethyl $n$-butyrate $(\boldsymbol{E}, \boldsymbol{F})$ in 20 -week-old mice. In control animals, several glomeruli distributed over the anterior half of the dorsal $O B$ show strong and differential activation by odorants, as indicated by the dark ISI signal $(\boldsymbol{A}, \boldsymbol{C}, \boldsymbol{E})$. In contrast, no discernible glomeruli are activated in $G \gamma 8-\mathrm{M} 72$ mice; instead a broad area of activation is detected in the anterior of the $O B(B, D, F)$. Scale bar, $250 \mu \mathrm{m}$. Medial (M) and anterior (A)

glomeruli composed of fibers expressing a single ntOR and thus results in the generation of mixed glomeruli. In fact, a positive correlation between the number of OSNs expressing a particular OR and convergence to a single glomerulus has been previously 
described (Ebrahimi and Chess, 2000; Feinstein and Mombaerts, 2004; Feinstein et al., 2004; Zou et al., 2004). Second, the formation of abnormally large G $\gamma 8$-tgOR glomeruli may partially disrupt normal cues required for accurate targeting of ntOR-OSNs. Finally, it is possible that some OSNs switch from expressing a $\mathrm{G} \gamma 8$-tgOR to expressing an ntOR after synaptic connections have been established, leading to mixed glomeruli.

\section{Concluding remarks}

Mammalian evolution has generated a vast family of G-protein coupled receptor genes that encode the odorant receptors (Buck and Axel, 1991; Zhang and Firestein, 2009). Remarkably, these genes and proteins have multiple functions in the OSN, setting odor selectivity (Malnic et al., 1999), guiding targeting to stereotypic locations in the olfactory bulb (Mombaerts et al., 1996; Wang et al., 1998), and restricting the expression of other ORs (Nguyen et al., 2007; Fleischmann et al., 2008). By manipulating the timing of OR gene expression, we have shown that both gene regulation (Nguyen et al., 2007) and OSN targeting can be dramatically altered. Together, our data demonstrate that OSNs must normally regulate the timing of expression of OR genes to ensure appropriate neural targeting.

\section{References}

Barnea G, O’Donnell S, Mancia F, Sun X, Nemes A, Mendelsohn M, Axel R (2004) Odorant receptors on axon termini in the brain. Science 304:1468.

Belluscio L, Katz LC (2001) Symmetry, stereotypy, and topography of odorant representations in mouse olfactory bulbs. J Neurosci 21:2113-2122.

Belluscio L, Gold GH, Nemes A, Axel R (1998) Mice deficient in G(olf) are anosmic. Neuron 20:69-81.

Bozza T, Feinstein P, Zheng C, Mombaerts P (2002) Odorant receptor expression defines functional units in the mouse olfactory system. J Neurosci 22:3033-3043.

Buck L, Axel R (1991) A novel multigene family may encode odorant receptors: a molecular basis for odor recognition. Cell 65:175-187.

Bulfone A, Wang F, Hevner R, Anderson S, Cutforth T, Chen S, Meneses J, Pedersen R, Axel R, Rubenstein JL (1998) An olfactory sensory map develops in the absence of normal projection neurons or GABAergic interneurons. Neuron 21:1273-1282.

Cheng LE, Reed RR (2007) Zfp423/OAZ participates in a developmental switch during olfactory neurogenesis. Neuron 54:547-557.

Chess A, Simon I, Cedar H, Axel R (1994) Allelic inactivation regulates olfactory receptor gene expression. Cell 78:823-834.

Ebrahimi FA, Chess A (2000) Olfactory neurons are interdependent in maintaining axonal projections. Curr Biol 10:219-222.

Feinstein P, Mombaerts P (2004) A contextual model for axonal sorting into glomeruli in the mouse olfactory system. Cell 117:817-831.

Feinstein P, Bozza T, Rodriguez I, Vassalli A, Mombaerts P (2004) Axon guidance of mouse olfactory sensory neurons by odorant receptors and the beta2 adrenergic receptor. Cell 117:833-846.

Fleischmann A, Shykind BM, Sosulski DL, Franks KM, Glinka ME, Mei DF, Sun Y, Kirkland J, Mendelsohn M, Albers MW, Axel R (2008) Mice with a "monoclonal nose": perturbations in an olfactory map impair odor discrimination. Neuron 60:1068-1081.

Hoon MA, Adler E, Lindemeier J, Battey JF, Ryba NJ, Zuker CS (1999) Putative mammalian taste receptors: a class of taste-specific GPCRs with distinct topographic selectivity. Cell 96:541-551.

Iwema CL, Schwob JE (2003) Odorant receptor expression as a function of neuronal maturity in the adult rodent olfactory system. J Comp Neurol 459:209-222.

Lin DM, Wang F, Lowe G, Gold GH, Axel R, Ngai J, Brunet L (2000) Formation of precise connections in the olfactory bulb occurs in the absence of odorant-evoked neuronal activity. Neuron 26:69-80.

Malnic B, Hirono J, Sato T, Buck LB (1999) Combinatorial receptor codes for odors. Cell 96:713-723.

Marks CA, Cheng K, Cummings DM, Belluscio L (2006) Activitydependent plasticity in the olfactory intrabulbar map. J Neurosci 26 : 11257-11266.

Mombaerts P, Wang F, Dulac C, Chao SK, Nemes A, Mendelsohn M, Edmondson J, Axel R (1996) Visualizing an olfactory sensory map. Cell 87:675-686.

Munger SD, Lane AP, Zhong H, Leinders-Zufall T, Yau KW, Zufall F, Reed RR (2001) Central role of the CNGA4 channel subunit in $\mathrm{Ca} 2+-$ calmodulin-dependent odor adaptation. Science 294:2172-2175.

Nguyen MQ, Zhou Z, Marks CA, Ryba NJ, Belluscio L (2007) Prominent roles for odorant receptor coding sequences in allelic exclusion. Cell 131:1009-1017.

Olsen SR, Wilson RI (2008) Lateral presynaptic inhibition mediates gain control in an olfactory circuit. Nature 452:956-960.

Potter SM, Zheng C, Koos DS, Feinstein P, Fraser SE, Mombaerts P (2001) Structure and emergence of specific olfactory glomeruli in the mouse. J Neurosci 21:9713-9723.

Ressler KJ, Sullivan SL, Buck LB (1994) Information coding in the olfactory system: evidence for a stereotyped and highly organized epitope map in the olfactory bulb. Cell 79:1245-1255.

Rogers KE, Dasgupta P, Gubler U, Grillo M, Khew-Goodall YS, Margolis FL (1987) Molecular cloning and sequencing of a cDNA for olfactory marker protein. Proc Natl Acad Sci U S A 84:1704-1708.

Rubin BD, Katz LC (1999) Optical imaging of odorant representations in the mammalian olfactory bulb. Neuron 23:499-511.

Ryba NJ, Tirindelli R (1995) A novel GTP-binding protein gamma-subunit, G gamma 8 , is expressed during neurogenesis in the olfactory and vomeronasal neuroepithelia. J Biol Chem 270:6757-6767.

Sullivan SL, Bohm S, Ressler KJ, Horowitz LF, Buck LB (1995) Targetindependent pattern specification in the olfactory epithelium. Neuron 15:779-789.

Tirindelli R, Ryba NJ (1996) The G-protein gamma-subunit G gamma 8 is expressed in the developing axons of olfactory and vomeronasal neurons. Eur J Neurosci 8:2388-2398.

Vassar R, Chao SK, Sitcheran R, Nuñez JM, Vosshall LB, Axel R (1994) Topographic organization of sensory projections to the olfactory bulb. Cell 79:981-991.

Wang F, Nemes A, Mendelsohn M, Axel R (1998) Odorant receptors govern the formation of a precise topographic map. Cell 93:47-60.

Zhang X, Firestein S (2009) Genomics of olfactory receptors. Results Probl Cell Differ 47:25-36.

Zou DJ, Feinstein P, Rivers AL, Mathews GA, Kim A, Greer CA, Mombaerts P, Firestein S (2004) Postnatal refinement of peripheral olfactory projections. Science 304:1976-1979. 